criterion of an association may be attributed to the relative paucity of the northern floras, rather than to the minute accuracy of their quadrat method. With the richer flora of the Alps other methods may be desirable, for different types of vegetation often require different methods of study. It is the opportunity afforded to workers from different countries of discussing and comparing such methods under field conditions, which constitutes one of the chief benefits to ecology of periodic international conferences such as the Phytogeographical Excursion which took place in Switzerland last summer.

In the discussions of recent years regarding the status of the "plant-association" as the primary unit of vegetation, and the methods of recognising and delimiting associations, there has been perhaps some tendency to lose sight of the physiological significance of the fact of association. It is customary to speak of " open " and " closed " plant-associations; but association in the strict sense can only be said to begin when the associated organisms exercise some influence on one another. In this sense some so-called open associations are not associations at all, at least in the initial pioneer stage. From the loose chance relations obtaining in the earlier stages, association gains in definiteness and importance as the vegetation becomes more and more closed. In other words, physical factors have a predominating influence in the earlier phases of development of vegetation, biotic factors becoming increasingly important in the later stages. While in general recognising these principles, Dr. Frey, dealing as he does mainly with vegetation of an open character, might have brought them out more clearly. For example, while agreeing in part with Oettli that association plays a less important rôle in the rock flora than elsewhere, he states that the occurrence of vascular rock plants is so sporadic that only the total list of species found in a wide region can represent the association. This usage, not at all uncommon, of the term association to denote something from which the element of real association is, to all intents and purposes, absent, can scarcely be justified.

Considerable attention was devoted to the study of succession on recent soils below the ends of glaciers, in the hope that light might be thrown on the problem of the relations of vegetation during the Glacial Period. The author finds the evidence somewhat conflicting, and exercises commendable caution in drawing conclusions. The most definite result arrived at is that apparently the unfavourable influence of the proximity of glaciers extends to a greater distance in a humid than in a dry climate. So far as it goes, this affords some support to Brockmann's hypothesis, which seeks to correlate the phenomena of the Glacial Period with an oceanic type of climate.

Dr. Frey's monograph is a good example of the thorough vegetational surveys which are being carried on so effectively at the present time in Switzerland. It is illustrated by well-reproduced photographs, and by transects and a coloured map. In the transects an effective mixture of symbols and initial letters is employed; the clearness of the map is somewhat interfered with by shading. Not the least useful feature is the very full and clear explanation in chap. iv. of the terms used.

\title{
The Radiation of Light by Excited Atoms.
}

PROF. G. MIE, in a paper in the Annalen der Physik for January, continues the development of a theory of the radiation process, which he outlined in the same journal in I92I. On the RutherfordBohr theory of atomic constitution, radiation takes place when an electron "falls" from an outer stationary orbit to an inner one, and several authors have regarded the emission of light by the atom as an almost instantaneous process, the energy being suddenly transferred to the ether, forming a radiation centre from which it travels out in waves, the frequency of which is given by $\mathrm{E}=h \nu$. The facts of interference make it necessary to assume that this ether centre goes on vibrating several million times before it comes to rest, which means, of course, an appreciable time interval, during the greater part of which the atom is supposed to be in the lower stationary state, i.e. the electron is revolving in the lower stationary orbit.

Prof. Mie supposes that the radiation is gradually given out by the atom to the ether while the alteration from one stationary state to another takes place. Thus the atom passes in a finite time through a constant succession of non-stationary states, the orbit of the electron altering gradually in a kind of contracting spiral from the outer to the inner Bohr orbit.

In the case of a freely radiating atom, Prof. Mie assumes that the fading out of the radiation ( $A b$ klingung) follows an exponential law; he formerly assumed that when the atom leaves the higher stationary state the radiation begins weakly, reaches a maximum at a certain distance from that state, and then drops exponentially; he now considers that it is quite possible that the intensity starts at once with a high value, and drops exponentially from the start. The essential difference between

$$
\text { NO. } 2842 \text {, VOL. I I } 3 \text { ] }
$$

the non-stationary electron orbits and the stationary is that in the former energy is given up by radiation the non-stationary movements are not periodic, and they do not obey the simple laws of classical mechanics.

In explaining the statistical equilibrium of a heated gas, it is necessary to assume the existence of two kinds of collisions between atoms, or between atoms and electrons. In collisions of the first kind the atom passes suddenly from a lower to a higher stationary state, the energy needed being derived from the kinetic energy of the colliding bodies; in a collision of the second kind, the atom passes from a higher to a lower state, and the energy given up is converted into kinetic energy. It is necessary to assume that similar collisions of the first and second kind may take place when an atom is in a non-stationary state, the essential condition being that the final result is always a stationary state of the atom.

Prof. Mie investigates the experimental results of R. W. Wood and of G. Cario on the decrease of light emission in a metallic vapour, excited by optical resonance or by an electron stream, when a neutral gas is added. Cario explained this by assuming that the time V (Verweilzeit) during which the atom remains in the upper stationary state is finite, while Mie shows that the same result follows if $\mathrm{V}=0$, and the time A (Abklingungszeit) during which the atom passes through the non-stationary states, is finite. $\mathrm{He}$ also shows that it is possible to assume that both the times $\mathrm{V}$ and $\mathrm{A}$ are finite, and takes into account a second possibility of sudden alterations in the atom, due to absorption of radiation. The photoelectric emission of electrons, when light of short wave-length and X-rays are absorbed, cannot be explained by a continuous absorption process, 
and Prof. Mie assumes, in order to get over the difficulty, that a kind of latent energy can be stored up in the atoms, or perhaps in the electrons, when suitable radiation falls upon them. It is shown that Planck's formula for "black" radiations follows from the theory on certain assumptions, using Einstein's method of proof.

\section{L'Enrégistreur Gueugnon.}

$\mathrm{IT}^{\mathrm{T}}$ is possible that there is a considerable amount of optimism in the declaration of M. Gueugnon, ". . . le graphique frappe les yeux de tous et non de quelques privilégiés. Chacun sait live un dessin." It is nevertheless a fact that the use of graphical methods as a means of making more real the results of analytical investigations is universally invoked by teachers, and even by the serious researcher when he desires to see concretely what his calculations lead him to. Most of us, however, are contented with rough-and-ready graphical presentations. When we have to show the effect of, say, two oscillations in different directions with various relationships between the periods, we just sketch the results roughly: the teacher who aims at greater clearness gives perhaps some detailed account of the way to plot the actual

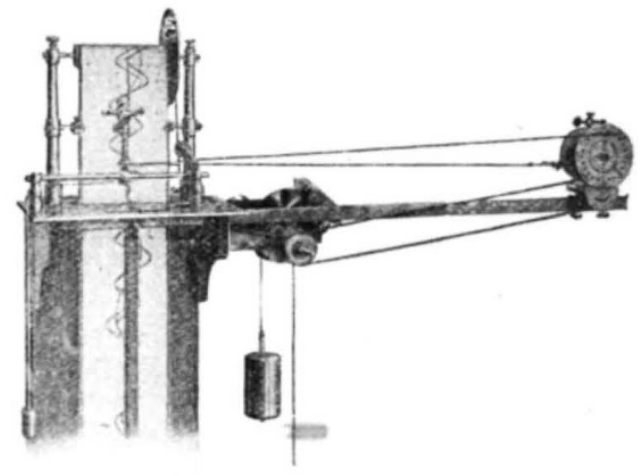

FIG. I.-The Gueugnon curve-tracer.

curves, and even instructs his pupils to draw a few for themselves. The results are not always encouraging.

$M$. Gueugnon is of opinion that the accurate drawing of the curves obtained in this or in any other type of problem is of great importance, as being the best means of obtaining at a single glance a view of all the variations involved. But he goes further and affirms that the student should have at his disposal a mechanical means of studying graphically the effects produced by all sorts of combinations of motions. It is for this reason that he has devised the instrument (Fig. I) which is the subject of this note. The principle is very simple, and one that the student will easily appreciate. A roll of paper F (Fig. 2) moves on a horizontal axle $\mathrm{AB}$, while a platform $\mathrm{TT}^{\prime}$ ' can be made to move horizontally, carrying a style at $\mathrm{S}$, which marks the paper. By giving different types of motions to the paper and to the platform carrying the style, we get various kinds of relationships between the $x$ and $y$ co-ordinates indicated in the figure. The style is also connected with a pendulum so that it can be made to oscillate and register uniform time intervals. It is clear that if the style swings with the pendulum while the platform is at rest, and the axle carrying the paper is made to move as the result of unequal loading on the two sides of the pulley attached to the axle, then we get an Atwood's machine with all the facilities for studying at first hand the formulæ of uniformly accelerated motion. If the style is not in oscillation, but the platform is made to move with uniform speed, then we get the study of the projectile.

Without going into details, it is sufficient to state that each of the rectangular co-ordinates $x$ and $y$ can be made to change uniformly, or with uniform acceleration, or periodically. It will be at once appreciated that in this way a very large number of varieties

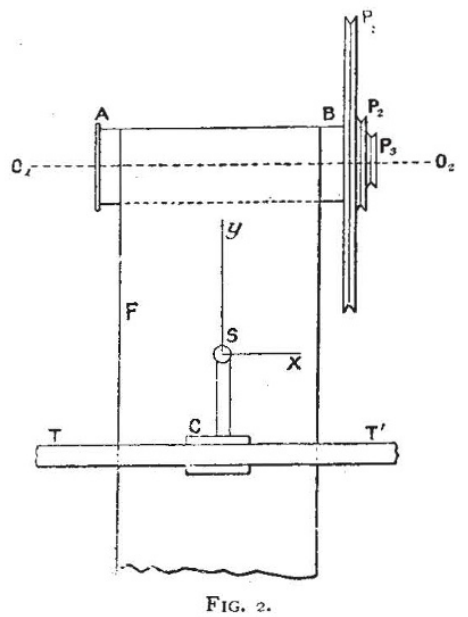

are obtained for the relation between $x$ and $y$, and the curves thus drawn can be made to illustrate most of the equations in the ordinary courses of pure mathematics and of mechanics and physics.

The writer has had the opportunity of inspecting a considerable number of curves thus obtained. The curves come out very clearly and neatly. One such curve is reproduced here (Fig. 3) as an illustration.

The enrégistreur has aroused the interest of the French Government, and a committee appointed by the Minister of Commerce and Industry in I9I 8 to report on the apparatus gave a unanimous verdict of approval and commendation. The Committee con-

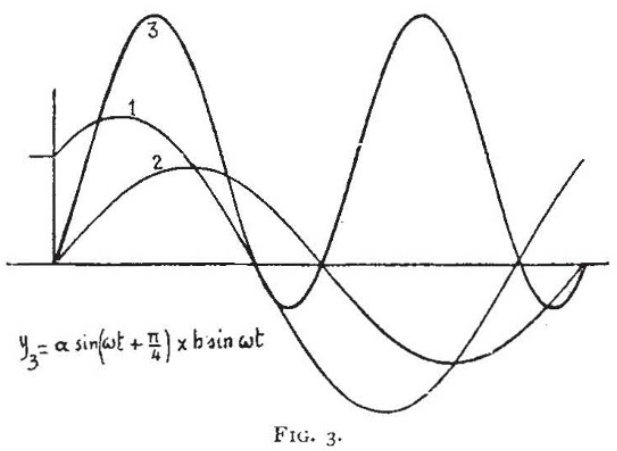

sidered that M. Gueugnon's apparatus is superior to those used hitherto, and that it facilitates and simplifies the study of mechanics, physics, and electricity. The apparatus has been actually introduced into the French National Schools of Arts and Crafts, into many institutions for scientific study in both France and Belgium, and into some universities.

The apparatus is manufactured by M. H. Morin of II, rue Dulong, Paris. The price is given as 2200 francs. The variations in the exchange rates make it difficult to translate this into English money with any accuracy, but it seems that the cost is something like $25 l$. N.O. 2842 , vOL. II 3 ] 